\title{
Analysis of Large Multi-modal Social Networks: Patterns and a Generator
}

\author{
Nan $\mathrm{Du}^{1}$, Hao Wang ${ }^{1}$, and Christos Faloutsos ${ }^{2}$ \\ 1 Nokia Research Center, Beijing \\ \{daniel.du, hao.ui.wang\}@nokia.com \\ 2 Carnegie Mellon University, Pittsburgh \\ christos@cs.cmu.edu
}

\begin{abstract}
On-line social networking sites often involve multiple relations simultaneously. While people can build an explicit social network by adding each other as friends, they can also form several implicit social networks through their daily interactions like commenting on people's posts, or tagging people's photos. So given a real social networking system which changes over time, what can we say about people's social behaviors ? Do their daily interactions follow any pattern ? The majority of earlier work mainly mimics the patterns and properties of a single type of network. Here, we model the formation and co-evolution of multi-modal networks emerging from different social relations such as "who-adds-whom-as-friend" and "who-comments-on-whose-post" simultaneously. The contributions are the following : (a) we propose a new approach called EigenNetwork Analysis for analyzing time-evolving networks, and use it to discover temporal patterns with people's social interactions; (b) we report inherent correlation between friendship and co-occurrence in on-line settings; (c) we design the first multi-modal graph generator $x$ Socia $\sqrt{1}$ that is capable of producing multiple weighted time-evolving networks, which match most of the observed patterns so far. Our study was performed on two real datasets (Nokia FriendView and Flickr) with 100,000 and 50,000,000 records respectively, each of which corresponds to a different social service, and spans up to two years of activity.
\end{abstract}

Keywords: Social Network Analysis, Graph Generator, Multi-modal Networks.

\section{Introduction}

Research of real world complex networks, like social networks [24, biological networks 11, topology [15] of WWW and Internet raises many significant and important problems. What patterns do the human-to-human interactions follow in large-scale social networks? How can we use such patterns to facilitate existing applications, such as anomaly detection [1] [18] and collective classification [8], and make further innovations?

\footnotetext{
1 http://research.nokia.com/people/hao_ui_wang/index.html
} 
As a result of the widespread adoption of Web 2.0 technology, social networking sites or services(SNS) are becoming ubiquitous and penetrate into every corner of people's daily lives. In such systems, people often belong to multiple social networks because of different person-to-person interactions. For example, in Nokia FriendView(http://betalabs.nokia.com/apps/nokia-friend-view), Flickr(www.flickr.com), Facebook(www.facebook.com), eBay(www.ebay.com), LinkedIn(www. linkedin.com), and Twitter(www.twitter.com), they all provide the basic function that enables people to add each other as friends through their content and conversations, which contributes to the emergence of our first type of social network, namely, the "friend network" or the "buddy network".

In addition, they also allow people to participate in specific activities. In FriendView, we can comment on the posts written by our colleagues. In Flickr, we can tag the photos uploaded by our friends. In eBay, we can rate the products sold by our partners. As a consequence, interactions with people centered around content form another type of social network called the "comment network" or the "participation network" from such activities as "commenting-onposts", "tagging-photos" and "rating-products". Therefore, these two types of social networks describe different facets of the same social networking system. For each of them, recent research has reported fascinating patterns, like [26] or lognormal [7] or Double Pareto LogNormal (DPLN) distribution 25] for the degree, as well as small and shrinking diameter [20].

In this paper, we are interested in answering the following questions:

- Do human social interactions and behaviors follow any temporal pattern ? Is there any regularity inherent in the daily activities of individuals and groups? Can we use such patterns to make predictions of their future behaviors?

- Given a real social networking site, is there any correlation between the buddy network and the participation network ? For instance, can we infer the friendship between two people in buddy network according to the discrete observations of their co-occurrence in the participation network ?

- How can we produce an intuitive generator that will mimic the behaviors, and correlations of these networks within a real social networking site simultaneously ? Most existing generators try to mimic the skewed distribution of degree or weight of only a single network, and thus fail to incorporate the possible correlations with other networks. Here, we want a multi-modal graph generator, which should describe the way in which the different social networks discussed above could co-evolve over time through the local interactions and activities between individuals.

Answering these questions can have many practical applications. First, identifying meaningful patterns hidden in human activities contributes to classifying people into different groups according to the similarity of their social behaviors, based on which we can have a deep insight about the composition and evolution of the network they belong to. Discovering new patterns also helps to discard unrealistic graph models. Second, knowing the correlation between different social relations is good for us to design better systems that further expand the range of human interactions by offering particular friend or product recommendations 
according to specific user context. Finally, intuitive graph models are also vital for simulation studies of routing algorithms when it is hard or even impossible to collect large real data, for understanding how the macro and global patterns of networks can emerge through the micro and local interactions among people over time, and for compressing and summarizing the real networks by model parameters.

The paper is then organized as follows. Section 2 reviews related work. Section 3 presents our observed patterns. Section 4 describes the $x$ Social model in detail. Section 5 gives the conclusion.

\section{Related Work}

In this section, we mainly survey the various discovered properties of real world networks, and several well-known graph generators.

\section{$2.1 \quad$ Network Patterns}

Many interesting patterns that real graphs follow have been discovered in recent work like the power-law distribution of the number of messages(photos), power law comment distribution, power law interval distribution[16], power law degree distribution 26, power law edge-weight distribution 24], power law node-weight distribution [24, Snapshot Power Law(SPL) 22], Clique Participation Law(CPL) [13, Clique-Degree Power Law(CDPL) 13, Triangle Weight Law 13, Eigenvalue Power Law(EPL) 2, shrinking diameter 20, and oscillating connected component (GCC\&NLCC) 22]. These patterns are important for us to understand the static and temporal properties of real world networks, to identify authorities and subgroups, as well as to refine routing algorithms and recommendations. Moreover, they are also vital for eliminating unrealistic graph generators and guiding us to design better ones, because ideally a graph model should be able to mimic all these patterns as many as possible.

\subsection{Graph Generators}

Generally, the graph generators of recent literature can be mainly classified as emergent graph models, and generative graph models. The basic principle of emergent graph models is that the macro network properties should emerge from the micro interactions of nodes over time. This type of models include ErdösRényi(ER) model [14, small-world model [27, BA model [6], Copy model 9], Random Multiplication Model [9, Forest Fire model 20, 'butterfly' model [22, and 'RTG' model [2. [See [5] and 9] for a detailed review and discussion]. Recently, Goetz [16] also provides models to mimic the evolving and spreading mechanism of blog systems. Moreover, research from the fields of economics and game theory also brought utility-based models [17, 4, 12, 13] where each node tries to optimize a specific utility function, and the network structure can arise from the collective strategic activities of all the nodes. Generative graph models often assume a global mathematic rule and perform iterations of such rule 
recursively until the generated networks meet several properties of real networks. Such models include kronecker multiplication model [19] and tensor model [3].

In summary, the majority of earlier graph generators often focused on modeling some main properties of only one single network. For example, [27 6] 20] 22. are limited in trying to model unweighted networks, and cannot be generalized to weighted networks. Goetz[16] describes the evolving process of blogs, but fail to incorporate the weights. Although RTG 2 can generate weighted graphs, it still only focused on one single network. As to the generative models, they usually cannot mimic the micro mechanism of node and edge addition, which makes it hard for us to understand the inherent natural process of real networks. In contrast, our work not only considers to mimic most of the known patterns, such as generating weighted networks from local nodes' interactions, but also focuses on co-evolution of different networks simultaneously.

\section{Tools and Observations}

In this section, we seek to find patterns inherent in large-scale on-line social networking sites. We first give a preliminary description of Nokia FriendView and Flickr datasets, and then we present the proposed EigenNetwork analysis method, and the discovered CoParticipation Friendship Correlation pattern.

\subsection{Data Description}

The datasets that we have analyzed include the interaction records from Nokia FriendView, and Flickr. Nokia FriendView is a location-enhanced experimental microblogging application and service operated by Nokia Beta Labs from the beginning of November 2008 to the end of September 2009 when the service was finished. It allows users to post messages about their status and activities from GPS-enabled Nokia S60 phones or from the web. Any two users can add each other to their buddy list through email request and confirmation. The users can also comment on the status messages posted by the buddies in their social network. As a result, we use three different types of record, < usrID, msgID, postTime, length $>,<$ usrID, buddyID, addTime $>,<$ userID, msgID, commentTime, length $>$, to describe these actions respectively.

Here, the edge weight of buddy network is the total number of comment times between them. For the dataset, there are 34,980 users, 20,873 buddy links, 62,736 status messages, and 22,251 comments [10]. The unique feature of this dataset is that it has recorded a complete evolving process of a social networking site from the very beginning to the end, over the course of 11 months. The detailed records enable us to have a deep insight about the way that people interact with each other. In the Flickr dataset (where people can upload photos, add contacts, and comment on or tag photos), we use similar tuples as Friend View to describe the data which includes about 542,105 users, 46,668,661 contact links, $101,520,484$ photos, and 8,999,983 comments from 2005 to 2007. Because these datasets belong to different services, have different scales, and were collected 
from different time, the diversity of our data can thus be guaranteed. Notice we only use the encrypted user id in this study, and restrict our interest only in the statistical findings within the data.

\subsection{EigenNetwork Analysis}

While the activities and interactions where each of us is involved every day appear nearly random, intuition tells that there also seems to be some regular recurrence of patterns, especially when we take the temporal, spatial, and social context into consideration. For instance, we may check several emails, and see some news after arriving at the office in the morning. Then we might chat with our friends through instant messaging during the working hours, and in the evening, we might write blogs, make comments, upload photos, or even play on-line games. Since a social network is inherently the collection of people and their interactions, analyzing the temporal behaviors of individuals and subgroups can help us to have a deep insight about the overall composition of the entire network.

We formulate our approach as follows. Given graph $\mathcal{G}$, for $\forall e_{i j} \in \mathcal{E}(\mathcal{G})$, we characterize the temporal activity of all the edges by a two-dimensional $E \times D$ binary matrix $\mathcal{M}$, where $E=|\mathcal{E}(\mathcal{G})|$, and $D$ is the total number of days that graph $\mathcal{G}$ has been in study.

$$
\mathcal{M}(p, q)=\left[\begin{array}{ccccc}
0 & 0 & 1 & 0 & \ldots \\
0 & 1 & 0 & 0 & \ldots \\
1 & 0 & 1 & 1 & \ldots \\
\ldots & \ldots & \ldots & \ldots & \ldots
\end{array}\right]
$$

Therefore, the $p$ th row represents the behavior of a particular edge $e_{i j}$ spanning the $D$ days. On a specific day $q$, if node $v_{i}$ and $v_{j}$ has at least one interaction with each other, then $\mathcal{M}(p, q)=1$; otherwise $\mathcal{M}(p, q)=0$. We then do Singular Value Decomposition(SVD) on matrix $\mathcal{M}$ and it is factorized as

$$
\mathcal{M}=U \times \Sigma \times V^{T}
$$

where the columns of $D$-by- $K$ matrix $V$ form a set of orthonormal input basis vectors for $\mathcal{M}$, the columns of $E$-by- $K$ matrix $U$ form a set of corresponding orthonormal output basis vectors, and the diagonal values in $K$-by- $K$ matrix $\Sigma$ are the singular values arranged in the descending order by which each corresponding input is multiplied to give a corresponding output.

By intuition, the $S V D$ on matrix $\mathcal{M}$ implicitly decomposes the $E$ edges into $K$ groups. Each column (or singular vector) $i$ of the $E$-by- $K$ matrix $U$ describes the extent to which each edge of $\mathcal{G}$ participates in the $i$ th group. Every column $j$ of the $D$-by- $K$ matrix $V$ shows the extent to which the $j$ th group is active on each day. The nonnegative real numbers on the diagonal of the $K$-by- $K$ matrix $\Sigma$ indicates the strength of each group. For each singular value $s_{i}$, the energy of $s_{i}$ is defined as $s_{i}^{2}$, so we keep the first few strongest singular values whose sum covers $80-90$ percentile of the total energy. Here, we build matrix $\mathcal{M}$ for 


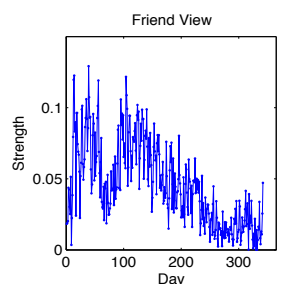

(a) 1 st vector

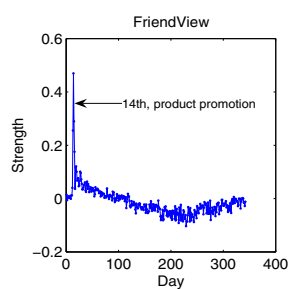

(b) 2nd vector

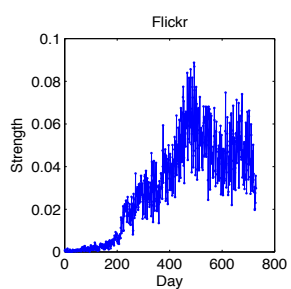

(c) 1 st vector

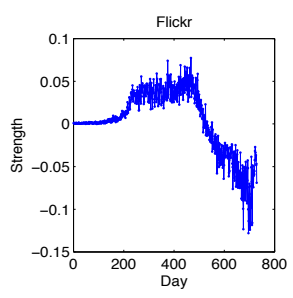

(d) 2nd vector

Fig. 1. The 1st and 2nd singular vector of matrix $V$ that describe the corresponding daily activities of the 1st and 2nd subgraph consisting of the selected edges in the participation network (formed by the comment relation) of FriendView (a-b), and Flickr (c-d) respectively

the participation network which emerges from the comment interactions among users in FriendView and Flickr respectively. $\mathcal{M}(p, q)=1$ means that for the $p$ th edge $e_{i j}$, at least one of the two nodes $\left(v_{i}\right.$ and $\left.v_{j}\right)$ commented on the messages or photos posted by the other one on the $q$ th day.

Figure 1 shows the top two singular vectors of the matrix $V$ from FriendView and Flickr. In Figure 11(a-b), we have two groups of edges that show different patterns of behavior. The first group of Figure 11(a) has basically a periodic pattern, while the second group of Figure 1(b) appears more bursty, where the spike occurs on the 14th day. Based on the complete records of FriendView, it was discovered that the 14th day was just during the week that Nokia did lots of advertising work to promote FriendView by calling for more open beta testers. For Flickr, both of the two groups shown in Figure 1(c-d) behave periodically. There is a clear trend of overall growth in the amplitude with some oscillation. We guess this may be caused by the quickly increased popularity and fast development of Flickr as more and more users joined in the system after the year 2006.

Figure 2 further presents the evolving process of the subgraph $G_{x}^{1}$ and $G_{x}^{2}$ consisting of the selected edges that actively participate in the 1 st and 2 nd singular vector of matrix $U$. Being active means that we only keep the set of edges whose sum of the energy (which is the square of the corresponding value) covers 80-90 percentile of the total energy. In Figure 2, the evolving pattern of $G_{x}^{1}$ and $G_{x}^{2}$ are clearly different. Subgraph $G_{x}^{1}$ contains a size- 4 clique (complete graph) where each blue-square node has connections with each other. This clique remains stable in topology and in total number of activities over the whole period, except for $G_{2}^{1}$ where five edges shown in red had significantly increased number of activities, and for $G_{3}^{1}$ where the the number of their activities dropped back. For $\forall e_{i j} \in \mathcal{E}\left(\mathcal{G}_{x}^{1}\right), x>1$, red color of $e_{i j}$ indicates that its weight (which is the total number of times that node $v_{i}$ and $v_{j}$ interact with each other in the $x$ th month) is significantly higher than its previous value in graph $\mathcal{G}_{x-1}^{1}$, and green color means the reverse. We made further investigations into the egocentric subgraph of around such 4 blue-square nodes in the entire network. Their average degree, and node betweenness [24] are 39 and 0.42 respectively. Because degree, 

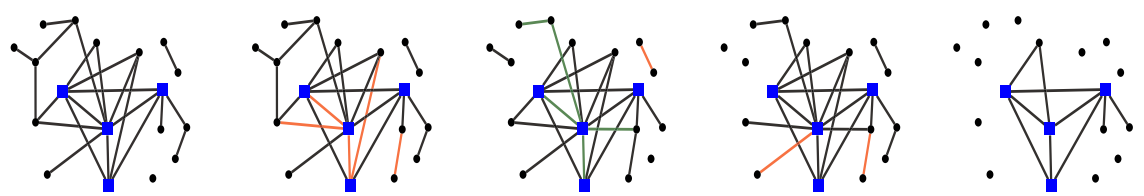

(a) $\mathcal{G}_{1}^{1}$ of 2008.11

(b) $\mathcal{G}_{2}^{1}$ of 2009.1
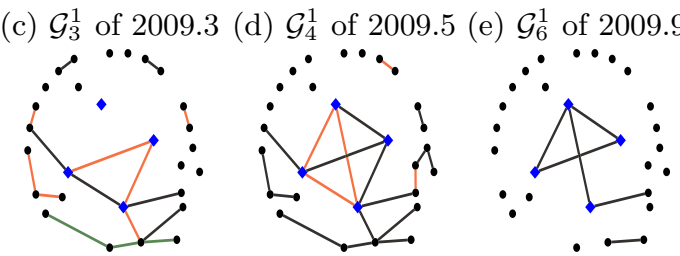

(f) $\mathcal{G}_{1}^{2}$ of 2008.11

(g) $\mathcal{G}_{2}^{2}$ of 2009.1

(h) $\mathcal{G}_{3}^{2}$ of 2009.3

(i) $\mathcal{G}_{4}^{2}$ of 2009.5

(j) $\mathcal{G}_{6}^{2}$ of 2009.9

Fig. 2. The evolving process of the subgraph $G_{x}^{1}$ and $G_{x}^{2}$ consisting of the selected edges belonging to the 1st(top row) and 2nd(bottom row) singular vector of matrix $U$ in the participation network of FriendView. $\forall G_{x}^{1}\left(G_{x}^{2}\right)$ where $x>1$, red indicates that the weight (representing the number of times that two users comment on each other's messages) is at least an order of magnitude higher than its previous value in $G_{x-1}^{1}$ $\left(G_{x-1}^{2}\right)$, green means the reverse, and black shows the same level.

and node betweenness are two popular measures to quantify a node's authority or centrality in a social network, the subgraph formed from these active edges in the 1st singular vector of matrix $U$ actually represents the central part or the core of FriendView's participation network.

We see that in November, 2008 and January, 2009, there are two significant increases in the number of interactions as most edges in the subgraph are red compared with the previous graph, which also coincides with the two spikes in Figure 1 (a). Moreover, because the open beta testing for FriendView actually finished in September, 2009, in Figure 2, the subgraph becomes sparse, when the interactions between users dropped gradually, and also conforms with the decreasing trend in Figure 1(a). In contrast, the subgraph $G_{x}^{2}$ is loosely connected. In the beginning, it only consisted of several separated edges. Notice in Figure 1(b), there is a bursty in the first month when Nokia did a lot of publicity work. As a result, there were many separated short-term interactions at that time.

Therefore, because subgraphs formed by the selected edges from the singular vectors of matrix $U$ (which are also the eigenvectors of $\mathcal{M} \times \mathcal{M}^{T}$ ) hold different local temporal patterns, and represent different compositions of the overall network, they are defined as the EigenNetworks, and our methodology is thus called EigenNetwork analysis.

Observation 1. EigenNetwork. The EigenNetworks can reveal local compositions of real world social networks, and hold different temporal patterns over time. 


\subsection{CoParticipation-Friendship Correlation}

In real social networking sites like FriendView or Flickr, on the one hand, people spend their daytime in following the updated status of their friends in the explicit buddy network. On the other hand, people are also the major players in the implicit participation network that emerges from the activities we adopt. As a consequence, is there any correlation between these two types of interaction ? Will the reoccurrence of one particular implicit activity contribute to a formation of the corresponding explicit interaction? More specifically, can we quantify the extent to which two people will become friends in the buddy network according to the discrete observations of their co-occurrences in the corresponding participation network? An underlying premise is that the probability for two people to become friends increases with the number of activities in which they have engaged together.

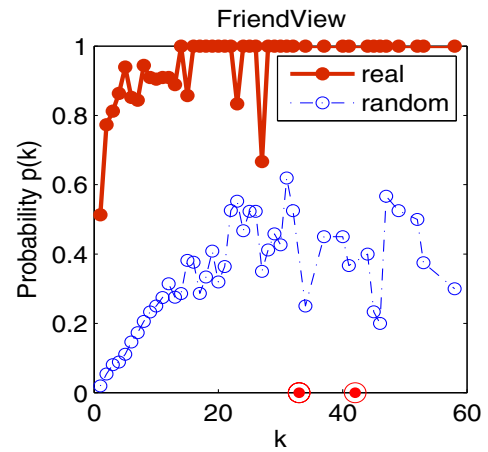

(a) Friend View

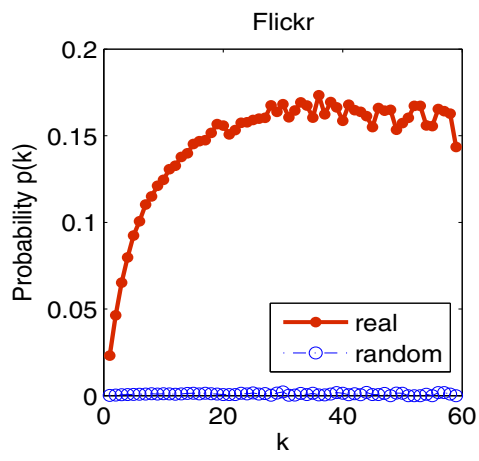

(b) Flickr

Fig. 3. The probability $P(k)$ of being friends as a function of the number of cocommented messages $k$ in Friend View (a), and photos in Flickr (b) respectively. For each $k$, red curve indicates the actual probability of being friends, and blue curve shows the expected value in random graphs. The outliers are marked by red circles.

Figure 3 shows this basic relationship in red color for FriendView and Flickr respectively, that is, the probability $P(k)$ of two people to become friends as a function of the total number of times that they have participated in $k$ common activities. $P(k)$ is calculated as follows. We first find all tuples $\langle i, j, k\rangle$ such that node $v_{i}$ and $v_{j}$ have $k$ participated activities in common. Then $P(k)$ is the fraction of such tuples for a given $k$ that node $v_{i}$ and $v_{j}$ are also friends in the buddy network. We see that when $k$ is roughly small $(k<30), P(k)$ has a strictly monotonic increase as $k$ increases. However, as $k$ becomes large, the marginal effect diminishes as $k$ increases.

Moreover, we would also like to evaluate how this empirical correlation compares to the corresponding result if comments were produced randomly with the same background distribution in real datasets. Specifically, for each node $v_{i}$, while we keep the number of posts(photos) on which she would comment the 
same as that in real dataset, we let her randomly choose among the posts(photos) this time. We then use $P_{0}(k)$ to denote the expected probability for a pair of nodes to become friends. If $P(k)>P_{0}(k)$, we say that the correlation is overrepresented in the data compared to chance; on the other hand, if $P(k)<P_{0}(k)$, then this correlation is underrepresented. To quantify the significance of $P(k)$ being over-or-underrepresented, we use the surprise 21] $S(k)$ which is defined as

$$
S(k)=\Delta(k) \times\left(P(k)-P_{0}(k)\right) / \sqrt{\Delta(k) \times P(k) \times(1-P(k))}
$$

where $\Delta(k)$ is the total number of tuples that have $k$ common posts(photos). $S(k)$ indicates the number of standard deviations by which the actual number of pairs being friends deviates from the expected number in random graphs. In Figure 3 , the plots in blue dash-line describe $P_{0}(k)$ vs. $k$ in random graphs for FriendView and Flickr respectively. According to the Cental Limit Theorem, the distribution of each $S(k)$ conforms approximately to a standard normal distribution, and it is expected on the order of tens to already be significant $(S(k)=6$ gives a $p$ value of $10^{-8}$ approximately) 21. However, in our datasets, we have found that the average $S(k)$ for $k<60$ and $P(k) \neq 0$ is 54.0 and 371.6 for FriendView and Flickr respectively, which is much larger and means that this correlation is statistically significant.

There are also some outliers(marked by red circles). The existence of such outliers means that although two people have engaged in many common activities together, they are still not friends yet. We guess this might be caused by users' ignorance or unawareness of each other. These types of users may only care about the messages or photos themselves by ignoring other people's comments at all. As a result, one possible application of the correlation shown in Figure 3 may be to help us with better recommendation systems, especially for the situation where $k$ is large, because as $k$ continuously increases, the number of pairs who have $k$ activities together decreases significantly, which makes it easier to give specific recommendations.

Observation 2. CoParticipation-Friendship Correlation(CPF). Given a real social networking site, the probability $P(k)$ of being friends for any pairwise persons increases with their $k$ activities in common. Although the marginal effect diminishes as $k$ increases, the effect remains significant.

\section{4 xSocial Model}

Next, we present our $x$ Social model where the " $x$ " means that it is a multi-modal graph generator that mimics real social networking sites to produce the buddy network and the participation network simultaneously. The guiding principle is that based on our understanding of existing patterns, we will devise a set of simple rules that each user would follow, and the entire social network will arise and evolve through the local interactions between individuals over time. Notice that this is actually a very challenging task, because the majority of prior work mostly focused on modeling only a single network. Our work is different as all 
the synthetic networks generated by $x$ Social should follow both the old and the new patterns mentioned in the previous section.

\subsection{Model Description}

$x$ Social model consists of the following four essential components:

- It is designed by using agent-based modeling approach. We have a set $\mathcal{A}$ of $n$ distinct agents, each of which has a preference value $f_{i}$.

- At each time, every agent performs three independent actions(write a message, add a friend and comment on a message) guided by the one-dimensional random walk mechanism.

- An agent chooses his friends either by their popularity or by the number of messages on which they have commented together, which is determined by his preference $f_{i}$.

- An agent can also follow the updated status of his friends by putting comments on the corresponding newly written messages.

Preference Value. For any agent $a_{i} \in \mathcal{A}, f_{i} \in(0,1)$ represents two different behaviors of people while they are using on-line social networking services. $f_{i}$ approaching to 1 means the agent likes to follow those active agents who have already written many messages, and continuously put new messages, while $f_{i}$ close to 0 indicates that he is interested in and pays more attention to the comments put by other agents.

Random Walk. In every step, each agent does a random walk on a line, and then chooses to write a message, or add a friend, or comment on a message whenever the walk returns to the origin (at state 0). We use three integers: $S_{w}$, $S_{a}$, and $S_{c}$ to represent the state of the corresponding action respectively. The initial state of $S_{w}, S_{a}$, and $S_{c}$ is 0 . For each variable, there are two types of transition. An agent $a_{i}$ adds or subtracts 1 from the variable's current state with probability $p_{i}$ and $1-p_{i}$ respectively. The agent $a_{i}$ performs the corresponding action whenever $S_{w}, S_{a}$, or $S_{c}$ returns to 0 again. Newman [23] shows that the inter-posting times follow a power-law distribution with exponent -1.5. Intuitively, the random walk reflects how frequently an agent uses the social networking service. On the one hand, when the probability $p_{i}$ approaches to 0 or 1 , the agents may just use the system in the very beginning, and never come back, just like that most users only register an account for curiosity in the beginning, but almost seldom use the service later. On the other hand, when $p_{i}$ is near 0.5 , the corresponding agents are relatively active users who can successively use the service, although they may be distracted by some random events to the nearby state around the origin.

Add a friend. For $\forall a_{i} \in \mathcal{A}$, once $a_{i} . S_{a}$ hits zero, he decides to expand his buddy network by exploring more friends. With probability $f_{i}, a_{i}$ trusts word-ofmouth and chooses an agent $a_{j}$ proportionally with the number of messages she has written, because the user who has published many messages will naturally attract attention of others so that she can expand the number of her followers. 
Once she has more followers, she would probably like to publish even more messages. In the opposite case, with probability $1-f_{i}, a_{i}$ picks an agent $a_{k}$ proportionally with the number of messages on which they have put comments together.

Make a comment. For $\forall a_{i} \in \mathcal{A}$, when $a_{i}$ decides to comment on some other messages at the moment $a_{i} . S_{c}=0$, she prefers the candidates newly written by her friends, because for most social networking services, we can often receive a notification once any one of our friends has updated her status. Therefore, $a_{i}$ chooses the message proportionally with $\frac{\# \text { comments }+1}{\text { age }+1}$, where \#comments is the number of existing comments on such message, and age is the number of times since its publication. As a result, the newly written messages which already have many comments will be chosen with very high probability.

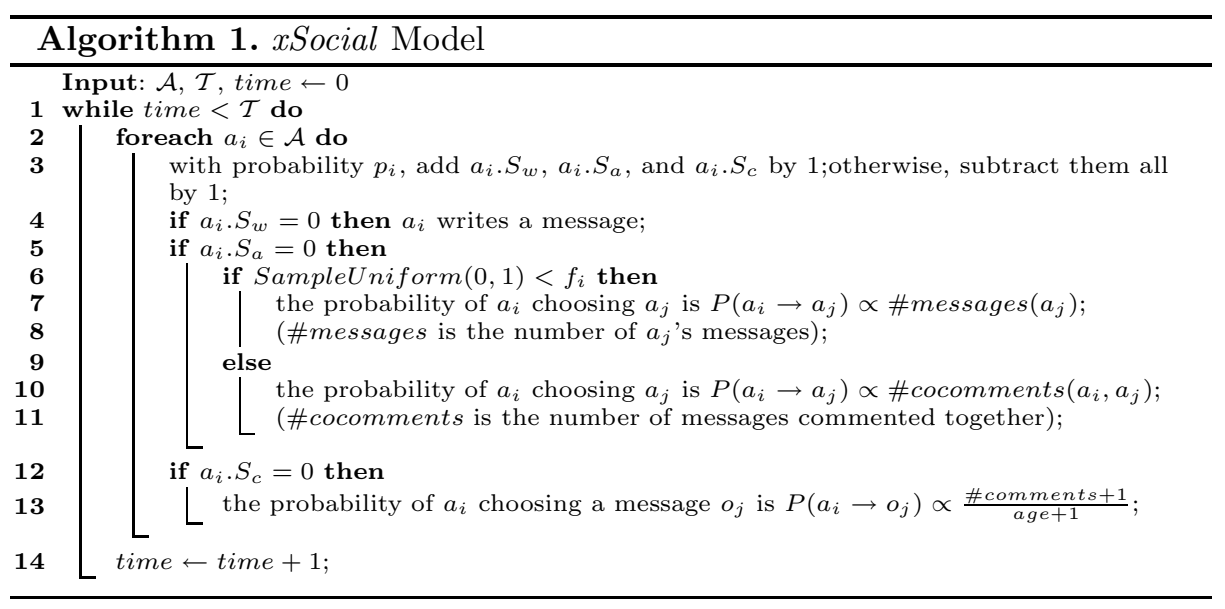

In summary, all these four major components in our xSocial model include very simple rules without assuming any prior sophisticated distributions or constraints. However, as we will show in the next section, both the buddy network and the participation network generated by this simple model can still match most patterns found on the real datasets. Pseudocode for $x$ Social is shown in algorithm 1.

\subsection{Model Analysis}

The $x$ Social model incorporates the interlinked evolving process of buddy network and participation network together, which is much more challenging to model jointly than separately. On the one hand, because the majority of existing graph generators mostly considers modeling a single type of network, there is no natural model to compare with our model. On the other hand, since the $x$ Social model also uses random walk to determine when to put a message or 

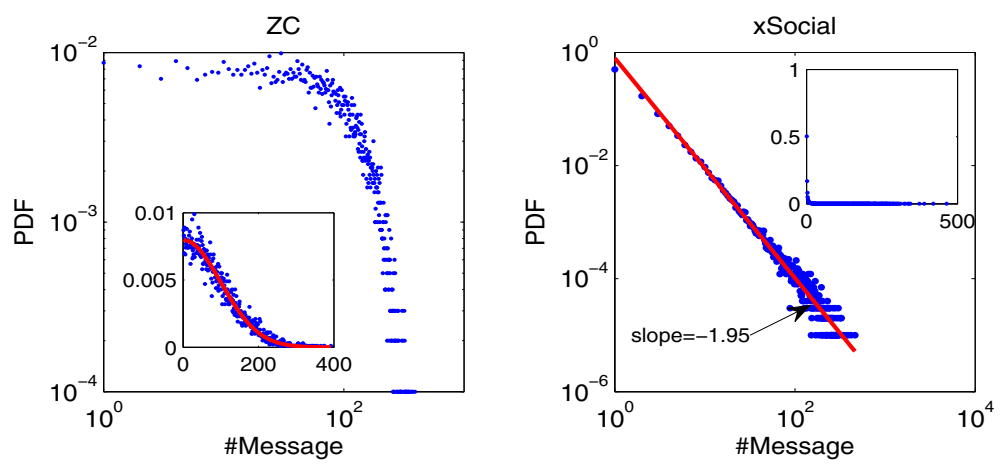

Fig. 4. PDF of \#Message. Left : ZC model gives a folded normal distribution $p(x) \propto$ $N_{f}(0, T)$ where $T$ is the number of times that random walk repeats; Right: xSocial model produces a power-law distribution $p(x) \propto x^{-1.95}$.

photo, for this point, we can at least make a comparison to the $Z C$ model [16]. However, as we will show as follows, even $Z C$ model still cannot give the correct distribution of the number of messages that users have posted. In Figure 4, $Z C$ model actually gives a folded normal distribution for people's posting behavior with 0 mean and $T$ deviation where $T$ is the number of times that random walk repeats. In contrast, our $x$ Social model matches the power law exponents well : -1.95 vs. -2.07 in Figure 6(m) and 6(q).

Because normal distribution and power-law distribution corresponds to two extreme cases : a homogeneous network, vs. a heterogeneous network, we believe that this difference arises as a result of the different random walk behaviors. In $Z C$ model, the probability for an agent to change his state is all set to 0.5, then each agent has equal opportunity to cross zero (make a post), although they may be distracted by some random events to the nearby state. However, this only models the behavior of active users who frequently use the system although they can be away from his computer for some random distractions. However, a real social networking site not only includes active users, but also involve lots of inactive users. These users just register an account for curiosity in the beginning, but seldom come back and use the system later. As a result, the probability of a random walk to change state in $x$ Social is designed to be different for each agent, which essentially enhances the system's heterogeneity. Because such heterogeneity significantly increases the model complexity, rigorous mathematical proofs are our current ongoing work.

\subsection{Model Validation}

How accurate is our model? A model is considered to be good if it is able to produce patterns and properties similar to those found in real world networks as many as possible. We simulated the model 15,000 times with 100,000 agents. For 


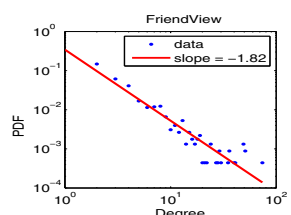

(a) Degree

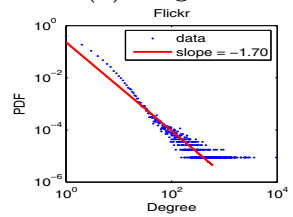

(f) Degree

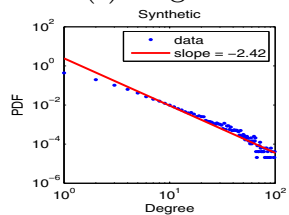

(k) Degree

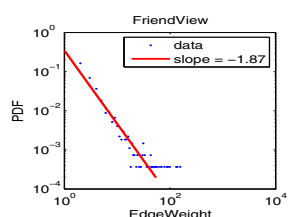

(b) EdgeWeight

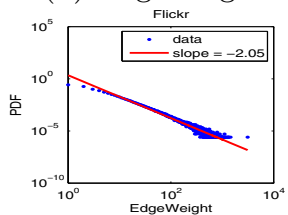

(g) EdgeWeight

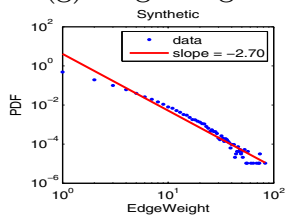

(l) EdgeWeight

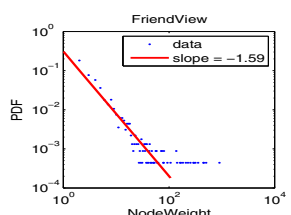

(c) NodeWeight

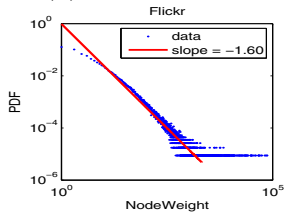

(h) NodeWeight

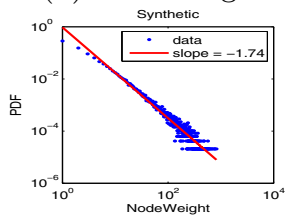

(n) NodeWeight

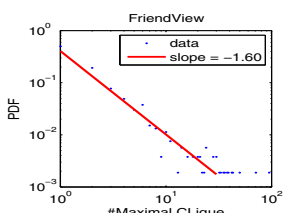

(d) CPL

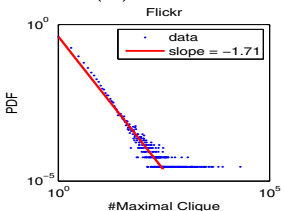

(i) CPL

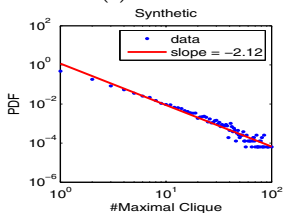

(o) CPL

Fig. 5. Comparison of the weighted buddy network, between the two real graphs (top two rows) for FriendView and Flickr, and our synthetic graph (bottom row)

each agent $a_{i}$, as $f_{i}$ and $p_{i}$ are independently and uniformly chosen at random from 0 to 1 , there is inherently no user-predefined parameters for $x$ Social to set.

We check with Edge-Weight Distribution[24], Node-Weight Distribution[24], as well as TWL[13 for weighted network, and Degree Distribution [26], SPL [22], CPL 13, CDPL 13, GCC\&NLCC 22, as well as EPL 2 for unweighted network. For each agent, we check with the distribution of the number of written posts, and the CoParticipation Friendship Correlation(CPF). For each post, we check with the distribution of the number of received comments.

Figure 5] and 6] show the related old and new patterns of the buddy network for Flickr and FriendView as well as for $x$ Social results, respectively. Figure 7 further compares the participation network (formed by the comment relation) between the real graph and the synthetic graph. Because we have similar observations on Flickr for the basic known patterns, here, we have to only present the results on FriendView in Figure 6 $(\mathrm{m}) \sim(\mathrm{p})$ and in Figure 7 due to the limit of space.

According to [22], there often exists a gelling point corresponding to the spike in the early stage of the growing network in Figure 6(s) and in Figure 7(h), after which several small connected components start to fuse into a giant connected component, and the 2nd and 3rd connected component remain constant size with small oscillations.

We see that the gelling point in Figure 6(o) and in Figure 7(d) is not as clear as in Figure 6(s) and in Figure 6(h), probably because the data was collected as a snapshot by month, and the data at the gelling point could be absorbed by 


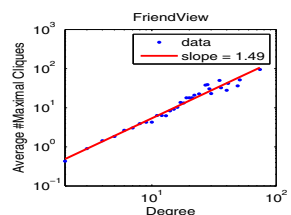

(a) CDPL

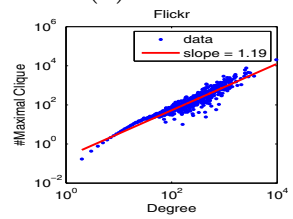

(e) CDPL

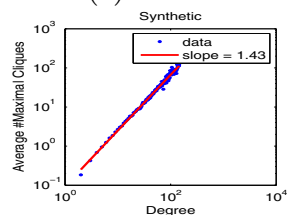

(i) CDPL

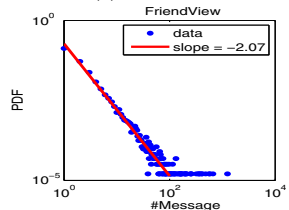

(m) Post

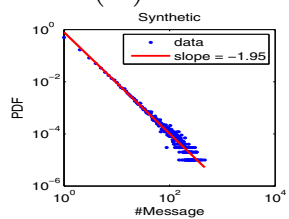

(q) Post

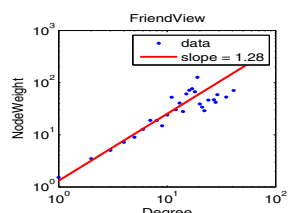

(b) SPL

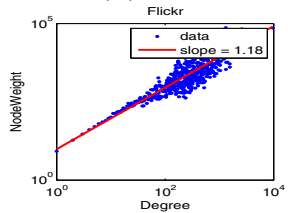

(f) SPL

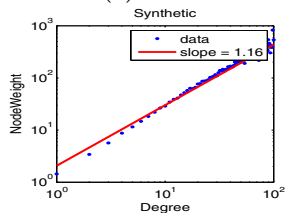

(j) SPL

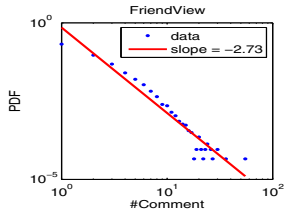

(n) Comment

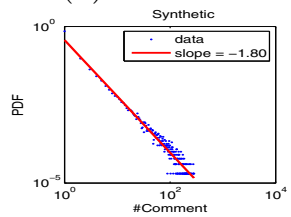

(r) Comment

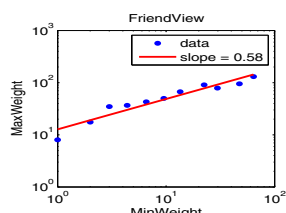

(c) TWL

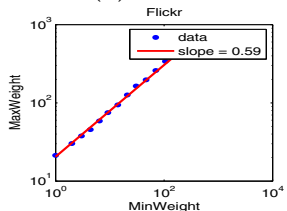

(g) TWL

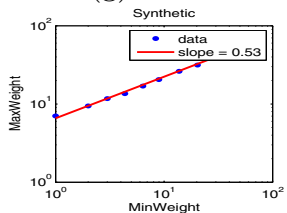

(k) TWL

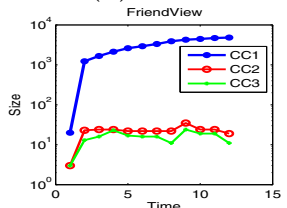

(o) GCC\&NLGCC

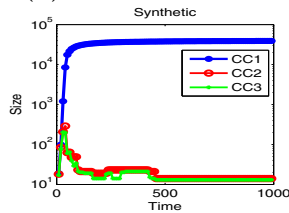

(s) GCC\&NLGCC

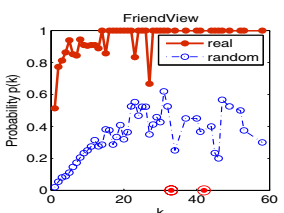

(d) $\mathrm{CPF}$

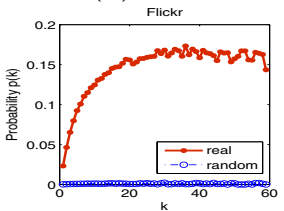

(h) $\mathrm{CPF}$

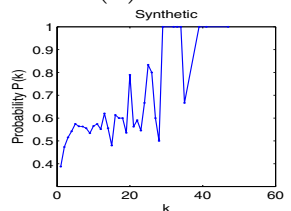

(1) $\mathrm{CPF}$

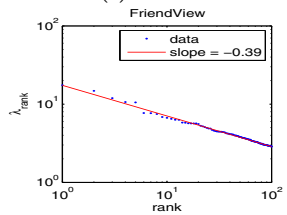

(p) EPL

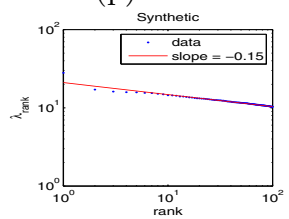

(t) EPL

Fig. 6. Comparison of the weighted buddy network cont'd, between the two real graphs from (a) to (h), and (m) to (p) for FriendView and Flickr, and our synthetic graphs from (i) to (l), and (q) to (t)

the later networks. By contrast, the synthetic networks are more fine-grained. However, after the gelling point, the size of the connected components keeps constant, showing that the synthetic networks coincide with the real networks. The effective diameter of the weighted buddy network and participation network of FirendView is approximately 9 and 10, while $x$ Social gives 9.7 and 9.5 respectively. In all cases, $x$ Social can give skewed distributions for both the buddy network and participation network which are remarkably close to the real ones. 


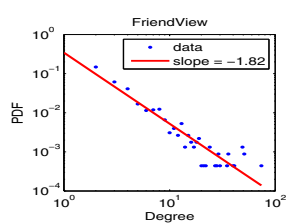

(a) Degree

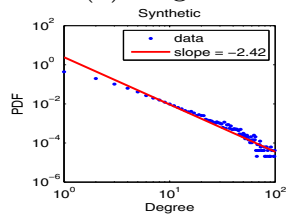

(e) Degree

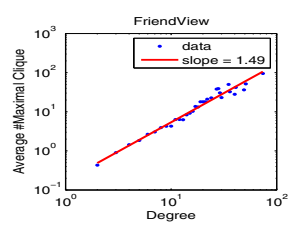

(b) CDPL

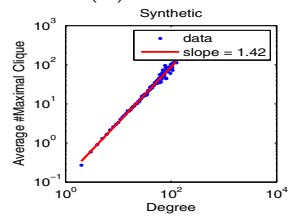

(f) $\mathrm{CDPL}$

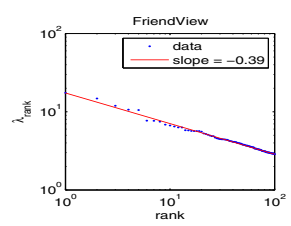

(c) EPL

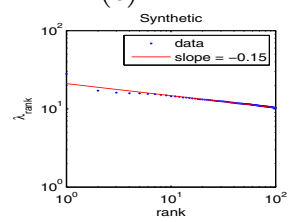

(g) EPL

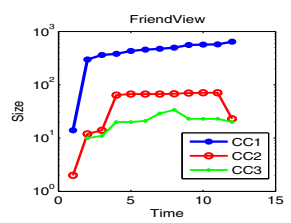

(d) Component

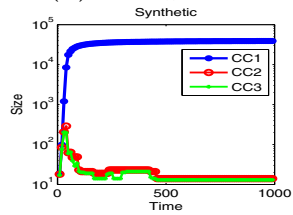

(h) Component

Fig. 7. Qualitative comparison of the comment network, between the real graph (top row), and our synthetic graph (bottom row)

\section{Conclusion}

We study multi-modal networks formed by friend and comment relations in two different datasets which have over 50 million records and span the course of 2 years. The main contributions are: (a) we proposed the EigenNetwork approach to analyzing time-evolving networks, and revealed that there exists temporal regularity with people's on-line social interactions; (b) we discovered inherent correlations between friendship and occurrence in on-line social networking settings; (c) we design the first multi-modal graph generator $x$ Social that stands out from the rest, because it does not include any user predefined parameters, it only uses local information, and it is capable of describing the co-evolving process of multiple weighted social networks that match the old and new patterns observed so far.

\section{References}

1. Aggarwal, C.C., Yu, P.S.: Outlier detection with uncertain data. In: SDM, pp. 483-493 (2008)

2. Akoglu, L., Faloutsos, C.: Rtg: A recursive realistic graph generator using random typing. In: PKDD, pp. 13-28 (2009)

3. Akoglu, L., McGlohon, M., Faloutsos, C.: Rtm: Laws and a recursive generator for weighted time-evolving graphs. In: ICDM, pp. 701-706 (2008)

4. Albers, S., Eilts, S., Even-Dar, E., Mansour, Y., Roditty, L.: On nash equilibria for a network creation game. In: SODA, pp. 89-98 (2006)

5. Albert, R., Barabasi, A.-L.: Statistical mechanics of complex networks. Reviews of Modern Physics (2002)

6. Barabasi, A.L., Albert, R.: Emergence of scaling in random networks. Science 286(5439), 509-512 (1999)

7. Bi, Z., Faloutsos, C., Korn, F.: The "DGX" distribution for mining massive, skewed data. In: KDD (August 2001), Runner up for Best Paper Award 
8. Bilgic, M., Getoor, L.: Effective label acquisition for collective classification. In: KDD, pp. 43-51 (2008)

9. Chakrabarti, D., Faloutsos, C.: Graph mining: Laws, generators, and algorithms. ACM Comput. Surv. 38(1) (2006)

10. Chin, A.: Finding Cohesive Subgroups and Relevant Members in the Nokia Friend View Mobile Social Network. CSE (4), 278-283 (2009)

11. Chung, F., Lu, L., Dewey, T.G., Galas, D.J.: J. Comput. Biol.10(5), 677-687 (2003)

12. Demaine, E.D., Hajiaghayi, M., Mahini, H., Zadimoghaddam, M.: The price of anarchy in network creation games. In: PODC, pp. 292-298 (2007)

13. Du, N., Faloutsos, C., Wang, B., Akoglu, L.: Large human communication networks: patterns and a utility-driven generator. In: KDD 2009, pp. 269-278 (2009)

14. Erdos, P., Renyi, A.: On the evolution of random graphs. Publ. Math. Inst. Hungary. Acad. Sci. 5, 17-61 (1960)

15. Faloutsos, M., Faloutsos, P., Faloutsos, C.: On power-law relationships of the internet topology. In: SIGCOMM, pp. 251-262 (August-September 1999)

16. Goetz, M., Leskovec, J., Mcglohon, M., Faloutsos, C.: Modeling blog dynamics. In: ICWSM 2009 (2009)

17. Laoutaris, N., Poplawski, L.J., Rajaraman, R., Sundaram, R., Teng, S.-H.: Bounded budget connection (bbc) games or how to make friends and influence people, on a budget. CoRR (2008)

18. Lee, J.-G., Han, J., Li, X.: Trajectory outlier detection: A partition-and-detect framework. In: ICDE 2008, pp. 140-149 (2008)

19. Leskovec, J., Chakrabarti, D., Kleinberg, J.M., Faloutsos, C.: Realistic, mathematically tractable graph generation and evolution, using kronecker multiplication. In: Jorge, A.M., Torgo, L., Brazdil, P.B., Camacho, R., Gama, J. (eds.) PKDD 2005. LNCS (LNAI), vol. 3721, pp. 133-145. Springer, Heidelberg (2005)

20. Leskovec, J., Kleinberg, J., Faloutsos, C.: Graphs over time: densification laws, shrinking diameters and possible explanations. In: KDD 2005, pp. 177-187 (2005)

21. Leskovec, J., Hunttenlocher, D., Kleinberg, J.: Signed Networks in Social Media. In: CHI, pp. 1361-1370 (2010)

22. McGlohon, M., Akoglu, L., Faloutsos, C.: Weighted graphs and disconnected components: patterns and a generator. In: KDD 2008, pp. 524-532 (2008)

23. Newman, M.E.J.: Power laws, pareto distributions and zipf's law. Contemporary Physics 46, 323 (2005)

24. Onnela, J.-P., Saramäki, J., Hyvönen, J., Szabó, G., de Menezes, A.M., Kaski, K., Barabási, A.-L., Kertész, J.: Analysis of a large-scale weighted network of one-toone human communication. New J. Phys. 9(6) (June 2007)

25. Reed, W., Jorgensen, M.: The double pareto-lognormal distribution - a new parametric model for size distribution (2004)

26. Watts, D.: Small Worlds:The Dynamics of Networks between Order and Randomness. Princeton University Press, Princeton (1999)

27. Watts, D.J., Strogatz, S.H.: Collective dynamics of 'small-world' networks. Nature 393(6684), 440-442 (1998) 